\title{
Comparison of Occlusive and Open Application in a Psoriasis Plaque Test Design, Exemplarily Using Investigations of Mapracorat $0.1 \%$ Ointment versus Vehicle and Reference Drugs
}

\author{
Walter Wigger-Alberti ${ }^{a}$ Ragna Williams ${ }^{a} \quad$ Yi-Ling von Mackensen $^{a}$ \\ Maciej Hoffman-Wecker ${ }^{a} \quad U^{\prime}$ ilke Grossmann ${ }^{b}$ Gerald Staedtler ${ }^{b}$ \\ Richard Nkulikiyinkab Kaweh Shakery ${ }^{b}$ \\ abioskin $\mathrm{GmbH}$, Hamburg, and ${ }^{\mathrm{b}}$ Bayer AG, Berlin, Germany
}

\section{Keywords}

Psoriasis plaque test - Efficacy · Echo-poor band .

Sonography $\cdot$ Clinical assessment

\begin{abstract}
Background/Aims: Psoriasis plaque tests (PPTs) are important tools in the early phases of antipsoriatic drug development. Two distinct PPT design variants (open vs. occluded drug application) are commonly used, but no previous work has aimed to directly compare and contrast their performance. Methods: We compared the antipsoriatic efficacy of mapracorat $0.1 \%$ ointment and reference drugs reported in 2 separate studies, representing open and occluded PPT designs. The drug effect size was measured by sonography (mean change in echo-poor band thickness), chromametry, and standardized clinical assessment. Results: Antipsoriatic effects were detectable for the study drugs in both occluded and open PPTs. Differences between the potency of antipsoriatic drugs and vehicle were observable. The total antipsoriatic effect size appeared to be higher in the occluded PPT than the open PPT, despite the shorter treatment duration (2 vs. 4 weeks). Effect dynamics over time revealed greater differences between some study drugs in the open PPT compared to the occluded PPT. Conclusion: Taking the higher
\end{abstract}

\section{KARGER}

(C) 2017 S. Karger AG, Basel

E-Mail karger@karger.com

www.karger.com/spp technical challenges for the open PPT into account, we recommend the occluded PPT as a standard screening setting in early drug development. In special cases, considering certain drug aspects or study objectives that would require procedural adaptations, an open PPT could be the better-suited design. Finally, both PPT models show clear advantages: classification as phase I studies, small number of psoriatic subjects, relatively short study duration, excellent discrimination between compounds and concentrations, parallel measurement of treatment response, and go/no go decisions very early in clinical development.

(c) 2017 S. Karger AG, Basel

\section{Introduction}

Plaque-type psoriasis is a common and mainly chronic skin disorder consisting of inflammation and hyperproliferation and presenting with scaly, erythematous, infiltrated, and often itchy skin lesions as a result of increased epidermal proliferation, impaired keratinocyte differentiation, dilated dermal blood vessels, and inflammatory cell infiltration into the skin [1]. Although recent findings point towards an autoimmune nature of psoriasis $[2,3]$, the underlying cause of the disease remains to

Walter Wigger-Alberti, MD

bioskin $\mathrm{GmbH}$

Burchardstrasse 17

DE-20095 Hamburg (Germany)

E-Mail walter.wigger@bioskinCRO.com 
be elucidated to date. Topical treatment of mild/moderate psoriasis remains a mainstay of antipsoriatic therapy, with a wide array of established topical drugs [4] as well as new, experimental topical drugs [5].

Other treatment options include phototherapy and systemic therapy with several drug classes, such as retinoids, corticosteroids, methotrexate, and fumaric acid esters, as well as anticytokine biologics, especially for severe psoriasis. The effectiveness of all these therapies is primarily evidenced by their ability to induce a measurable improvement in the cutaneous symptoms associated with psoriatic plaques. Assessment of the clinical treatment response is an accepted approach to investigate the efficacy of new agents for the treatment of plaque-type psoriasis; this is usually evaluated in confirmatory studies with drug administration from 8 up to 12 weeks. However, to optimize the clinical development program for topically applied drugs, it is of interest to obtain reliable data for the efficacy of potential antipsoriatic compounds early in the development process.

In 1972, Dumas and Scholtz [6] developed the psoriasis plaque test (PPT) to initially evaluate the potency of topical corticosteroids. Further modifications have been published since then to evaluate various types of topical antipsoriatic formulations. For an early identification of new potentially useful topical antipsoriatics, the PPT is helpful as a standardized proof-of-concept model which allows intraindividual comparisons of the clinical efficacy of multiple topical formulations in subjects with stable plaque-type psoriasis $[7,8]$. In the early 1980s, the validity of the PPT was confirmed for a variety of antiproliferative substances in an extensive multicenter study [9]. Katz et al. [10] demonstrated its capacity to differentiate between corticosteroids of different potency applied in different formulations and in different concentrations.

In the original PPT design by Dumas and Scholtz [6], the efficacy of topical corticosteroids for the treatment of plaque-type psoriasis was evaluated clinically following standardized occlusive application over several days. Whereas this design with simply grading of clearing is well suited for the evaluation of corticosteroids of varying potencies, it is not sensitive enough to differentiate formulations with identical active ingredients [11] or formulations of similar potency. Biophysical measurement methods have been added to the design for objective measurement of the inflammatory alterations accompanying psoriasis, greatly improving the sensitivity of the test $[12,13]$. One of the most important clinical signs in psoriasis is the extent of skin thickening due to acanthopapillomatosis. This phenomenon, together with

Comparison of Occlusive and Open

Psoriasis Plaque Tests the presence of inflammatory infiltration, can be measured by $20-\mathrm{MHz}$ sonography and is represented by a nonechogenic or echo-poor band (EPB), probably the most relevant outcome used as variable for antipsoriatic treatment effects [11]. Objectively capturing EPB as a surrogate for infiltrate thickness allows for greatly improved discrimination and sensitivity compared to clinical assessment. Using different frequencies of sonography, this method has been clinically and scientifically accepted since many years [12, 14-18]. Nevertheless, it has to be considered that sonography is a highly suitable bioengineering method to evaluate the potential activity in the PPT in an early phase of drug development, but final proof of antipsoriatic efficacy has to be studied in later phases.

Generally, clinical improvement is assessed as a secondary variable in the PPT. Because of the small size of the test fields and difficulties encountered in clinical assessment, separate clinical gradings of infiltration depth and erythema are not performed, rather an assessment of general improvement or worsening compared with the untreated plaques beneath the hydrocolloid dressing [11]. Scaling cannot be evaluated since descaling is a necessary condition for sonographic measurements. Therefore, the effect of antipsoriatic drugs on exaggerated proliferation could not be evaluated in the PPT model and has to be investigated in subsequent trials.

The PPT has been successfully performed in countless studies over the past years using a classic occlusive design in which the test areas were covered with various materials, for example Finn Chambers ${ }^{\circledR}[12,19,20]$, Foliodrape $^{\circledR}$ film $[21]$, or the originally used Saran Wrap ${ }^{\circledR}[6$, 22]. Few PPT studies have been published in which test products were applied in an open manner, protecting the test areas with nonocclusive gauze [23,24]. To our knowledge, no direct comparison of these two techniques using the same test products is available yet; however, it is plausible that data may differ due to differences in the pharmacodynamic effects in occlusive versus open drug application [7]. This was previously described for another test system, the vasoconstrictor assay, in which differences between occluded and open application were seen for several corticosteroid formulations [25]. Therefore, we here report the results of our evaluation of the antipsoriatic activity of mapracorat compared to the marketed topical antipsoriatic product prednicarbate $0.25 \%$ ointment in clinical experiments using the PPT both in the classic occlusive design and the nonocclusive design. Further comparisons were done versus vehicle in the occluded PPT and versus clobetasol $0.05 \%$ ointment, calcipotri-

Skin Pharmacol Physiol 2017;30:102-114 103 
ene $0.005 \%$ ointment, and calcipotriene $0.005 \% /$ betamethasone dipropionate $0.05 \%$ ointment in the open PPT.

Mapracorat, a selective glucocorticoid receptor agonist developed by Bayer Pharmaceuticals, is an investigational drug belonging to a new class of small molecules that is structurally unrelated to steroids, although similar in certain aspects of expected pharmacological action facilitated by binding to the glucocorticoid receptor. Selective glucocorticoid receptor agonists are an attractive class of compounds for the topical treatment of inflammatory skin diseases due to a potentially better effect/side effect profile compared to classical corticosteroids.

We compared mapracorat $0.1 \%$ ointment to marketed topical antipsoriatics using the PPT applied in the classical model using occluded test areas and, subsequently, in an open design without occlusion by evaluating efficacy measurements (EPB by sonography, clinical scoring, and erythema measurement by chromametry) with 3 main objectives: (1) to evaluate the reliability of each technique and its ability to discriminate between the antipsoriatic activity of different agents; (2) to examine whether there is consistency of observed results between the 2 techniques; and (3) to evaluate whether there are possible advantages of one design over the other.

\section{Methods}

Subjects

Two randomized, double-blind, controlled, clinical trials were conducted at bioskin $\mathrm{GmbH}$, Germany; the occluded PPT took place between August and October 2007 and the open PPT between February and May 2013. The studies were performed in compliance with local laws and regulations, the Declaration of Helsinki, and the International Conference on Harmonization Good Clinical Practice Guidelines. The study protocols were approved by the relevant independent ethics committee and national health authority.

In each study, 24 Caucasian subjects with stable plaque-type psoriasis were investigated after written informed consent had been obtained. All subjects were in otherwise good health, as determined by clinical and laboratory examinations at screening, and eligible for study participation, as assessed by predefined inclusion and exclusion criteria. All subjects were aged $\geq 18$ years and had plaques of adequate size which were not localized on the head and neck, elbows and knees, palms and soles, nails and folds, or other mechanically strained sites. Exclusion criteria included guttate, erythrodermic or pustular psoriasis, spontaneously improving or rapidly deteriorating plaque-type psoriasis, or severe recalcitrant psoriasis requiring additional therapy. Subjects who were pregnant or lactating or had underwent a therapy for psoriasis with any systemic treatment 3 months or any local treatment 2 weeks prior to screening were not allowed to participate.

\section{Test Designs}

For the occluded PPT, the area of the target plaques had to be sufficient for 4 test fields measuring $1.1 \mathrm{~cm}^{2}$ each (total treatment area $4.4 \mathrm{~cm}^{2}$ ). A somewhat greater area of target plaques was chosen for the open PPT with 5 test fields measuring $3 \mathrm{~cm}^{2}$ each (total treatment area $15 \mathrm{~cm}^{2}$ ), which was necessary to ensure appropriate drug application similar to a clinical use situation. Enough space was left between the test fields in both studies to exclude interactions with neighboring fields.

In both studies, the target plaques were pretreated with salicylic acid $5 \%$ in petrolatum 5 days prior to treatment initiation in order to remove scales. Twice daily applications with this pretreatment were continued at home for up to 1-2 days before treatment with the study drugs.

For exact localization, the border of the plaques and the exact localization of the test fields were recorded on a transparent foil prior to the first treatment on day 1.

Application of the study drugs was performed by study personnel once daily on 6 days a week for 2 weeks in the occluded PPT (12 applications in total) and for 4 weeks in the open PPT (24 applications in total). The test drug mapracorat $0.1 \%$ ointment was the same in both studies as well as the prednicarbate $0.25 \%$ ointment. In the open PPT, additional marketed topical antipsoriatic formulations were tested. All study drugs were provided by Bayer AG.

\section{Occluded PPT}

On day 1 (baseline), the test fields (1 per study drug plus 1 for an nontreated control) were defined on the previously determined target plaques. A template made from hydrocolloid dressings (Varihesive; Bristol-Myers Squibb, Munich, Germany) was drawn from the foil. Four holes corresponding to the size and location of the test fields determined were punched in the hydrocolloid dressings. The hydrocolloid dressing delineated the test fields and ensured their exact relocalization.

Of each study drug, $200 \mu \mathrm{L}$ (mapracorat $0.1 \%$ ointment, vehicle ointment without pharmaceutical active ingredient, and prednicarbate $0.25 \%$ ointment) were applied to 3 test fields using test chambers (Duhring chambers; Diehl Metal Applications, Berlin, Germany; $12 \mathrm{~mm}$ inside $\varnothing, 14 \mathrm{~mm}$ outside $\varnothing$, the distance between 2 chambers was at least $1.5 \mathrm{~cm}$ ) completely filling the test chamber. The 4th field was occluded with a Duhring chamber but left untreated and served as nontreated control. The Duhring chambers were seated in the respective holes punched in the hydrocolloid dressings, which were additionally fixed on the skin with adhesive patches (BSN, Hamburg, Germany). The chambers themselves were fixed in place with Leukosilk ${ }^{\circledR}$ (BSN, Hamburg, Germany) and were removed before each new application. Any remaining product residues were cleared by gently cleansing each test field with a separate soft tissue.

\section{Open PPT}

On day 1 (baseline), the test fields (1/study drug) were defined on the previously determined target plaques. The exact localization of each test field was confirmed at each visit using a stencil made from transparent foil during the test field determination prior to the first treatment on day 1 .

Of the respective preparation, $10 \mu \mathrm{L}$ (mapracorat $0.1 \%$ ointment was compared with 4 other drugs: prednicarbate $0.25 \%$ ointment, clobetasol $0.05 \%$ ointment, calcipotriene $0.005 \%$ ointment, 
and calcipotriene $0.005 \%$ /betamethasone dipropionate $0.05 \%$ ointment) were applied directly on the previously selected test field to cover the whole test field with a thin layer, mimicking a clinical use situation. The full amount was gently distributed on the test field separately for each treatment and test field with a finger sheltered with a saturated finger cot. The distance between 2 test fields was at least $2 \mathrm{~cm}$. After application, sufficient time was given for the study drugs to dry. To prevent mixing of the preparations during drying, the test fields were protected for at least 60 min with a piece of gauze, fixed with Leukosilk ${ }^{\circledR}$ in a nonocclusive way.

\section{Measurements/Assessments}

In both studies, target plaques were assayed in the same way by EPB thickness measurements using 20-MHz B-mode sonography (2-dimensional images), erythema measurement by chromametry, and clinical assessment of efficacy. The assessments and measurements were done prior to the application of new drug on days 3,5 , $8,10,12$, and 15 in the occluded PPT and on days $4,8,11,15,18$, 22, 25, and 29 in the open PPT.

EPB Thickness Measured by 20-MHz B-Mode Sonography

The EPB thickness was measured with a high-frequency 20$\mathrm{MHz}$ transducer of a sonography device (taberna pro medium, Lüneburg, Germany). If necessary, gentle descaling using a detergent was performed in order to enable appropriate sonography measurements.

Serial A scans were transformed digitally into 2-dimensional images of the skin (B mode). The B-mode image of a psoriatic plaque was characterized by the EPB located in the typical position, beneath the entry echo, and has previously been shown to correlate with the clinical severity of a psoriasis plaque $[15,16,18]$.

One scan was made per test field and time point. The width of the EPB was measured and recorded.

\section{Chromametry}

All measurements were performed with the same type of device (Chroma-Meter CR 400; Konica Minolta Inc., Munich, Germany). In accordance with the $\mathrm{L}^{*} \mathrm{a}^{*} \mathrm{~b}^{*}$ system, a decrease or increase in the $\mathrm{a}^{*}$ value (color range from green $[-]$ to red $[+]$ ) corresponded to a decrease or increase in the degree of erythema, respectively. Three measurements were taken from each field during each measurement series. The mean of the $3 \mathrm{a}^{*}$ values was calculated and served as a basis for statistical analysis.

\section{Clinical Assessment of Efficacy}

Clinical assessment of the test fields was performed by dermatologically trained evaluators using a 5-point score: -1 : worsened, 0 : unchanged (no effect), 1: slight improvement, 2: clear improvement but not completely healed, and 3: completely healed. In the occluded PPT, the comparison was made with the nontreated control, while in the open PPT, the comparison was made with the untreated plaque surrounding the test fields. Clinically apparent differences in erythema and infiltration contributed to this global assessment.

\section{Safety}

Adverse events were recorded and their nature and frequency of occurrence were assessed over the entire study period.
Statistics

In both tests, the occlusive and the open PPT, efficacy analysis was done for the full analysis set (FAS) and for the per protocol set (PPS). Safety analysis was performed for the safety analysis set.

The FAS included all subjects who were treated with at least 1 dose of the study drugs, and for whom at least 1 clinical assessment of efficacy observation after the start of treatment was available.

The PPS included all treated subjects who did not present any major protocol deviations or significant protocol deviation affecting the efficacy variables and who had completed the study.

The safety analysis set included all subjects who were treated with at least 1 dose of the study drugs.

In both studies, predefined explorative descriptive analyses were performed.

This publication focused on the comparison of occlusive and open application PPTs by comparing the mean absolute values as well as the mean absolute and percent changes from baseline in EPB on day 15 (both studies) and day 29 (open PPT). Results of the statistical testing of the pairwise comparisons of EPB changes versus baseline are presented for the occluded and open PPTs. Statistical tests were 2 -sided, and no adjustment for multiplicity was done. $p$ values and $95 \%$ confidence intervals for differences between treatments were determined within the framework of the statistical model (2-factorial analysis of variance).

For the results of chromametry, percent changes from baseline to day 15 (both studies) and day 29 (open PPT) are presented. For the clinical assessment, frequencies and percentages of scores are summarized.

\section{Results}

\section{Study Population}

In both tests, 24 subjects were studied: 24 men (mean age 49.1 years, range 30-76 years) and 4 women and 20 men (mean age 46.2 years, range 27-65 years) received blinded treatment with the study drugs applied to randomized test fields in the occluded and in the open PPT, respectively.

Data from all subjects were valid for planned analyses in both studies. In the PPS analysis, data from 24 and 23 subjects were valid in the open and the occluded PPT, respectively. One subject in the occluded PPT was excluded from the PPS due to a major protocol deviation (subject missed study day 14 for removal and new application of study drugs). All subjects completed the treatment period. Since the results of the FAS analysis were similar to those of the PPS analysis, only the results of the FAS are presented here.

\section{EPB Thickness Assessed by 20-MHz B-Mode} Sonography

\section{Occluded PPT}

The results of the sonography measurements showed clear and comparable reductions in mean EPB for mapra-

Comparison of Occlusive and Open

Skin Pharmacol Physiol 2017;30:102-114 
Table 1. Echo-poor band (EPB) thickness ( $\mu$ m; means \pm SD) measured using 20-MHz B-mode ultrasound in an occlusive $(n=24)$ and open $(n=24)$ psoriasis plaque test $(\mathrm{PPT})$ setting

\begin{tabular}{|c|c|c|c|c|c|}
\hline \multirow[t]{2}{*}{ Treatment } & \multirow[t]{2}{*}{ Baseline } & \multirow[t]{2}{*}{ Day 15} & \multirow[t]{2}{*}{ Day 29} & \multicolumn{2}{|c|}{ Change from baseline } \\
\hline & & & & day 15 & day 29 \\
\hline \multicolumn{6}{|l|}{ Occlusive PPT } \\
\hline Mapracorat $0.1 \%$ & $369.8 \pm 143.86$ & $113.3 \pm 75.87$ & - & $-256.6 \pm 122.50$ & - \\
\hline Prednicarbate $0.25 \%$ & $367.9 \pm 133.51$ & $89.5 \pm 99.63$ & - & $-278.4 \pm 79.26$ & - \\
\hline Vehicle & $362.0 \pm 121.14$ & $366.2 \pm 133.05$ & - & $4.2 \pm 45.40$ & - \\
\hline Nontreated control & & & - & & - \\
\hline (untreated, occluded test field) & $367.5 \pm 137.56$ & $376.6 \pm 139.90$ & & $9.1 \pm 14.39$ & \\
\hline \multicolumn{6}{|l|}{ Open PPT } \\
\hline Mapracorat $0.1 \%$ & $541.0 \pm 208.5$ & $427.4 \pm 214.4$ & $297.2 \pm 205.2$ & $-113.6 \pm 159.6$ & $-243.8 \pm 218.4$ \\
\hline Prednicarbate $0.25 \%$ & $555.7 \pm 231.8$ & $365.0 \pm 175.5$ & $281.3 \pm 202.4$ & $-190.7 \pm 165.6$ & $-274.4 \pm 204.9$ \\
\hline Clobetasol 0.05\% & $557.4 \pm 247.0$ & $188.3 \pm 152.1$ & $66.1 \pm 96.2$ & $-369.1 \pm 242.4$ & $-491.3 \pm 234.2$ \\
\hline Calcipotriene $0.005 \%$ & $566.5 \pm 233.4$ & $357.8 \pm 194.4$ & $307.9 \pm 193.0$ & $-208.7 \pm 212.9$ & $-258.6 \pm 201.3$ \\
\hline $\begin{array}{l}\text { Calcipotriene } 0.005 \% / \text { beta- } \\
\text { methasone dipropionate } 0.05 \%\end{array}$ & $552.8 \pm 223.1$ & $193.0 \pm 128.2$ & $96.1 \pm 115.9$ & $-359.7 \pm 189.1$ & $-456.7 \pm 216.3$ \\
\hline
\end{tabular}

Table 2. Mean changes from baseline in echo-poor band (EPB) thickness (\%) measured using 20-MHz B-mode ultrasound in an occlusive $(n=24)$ and open $(n=24)$ psoriasis plaque test (PPT) setting

\begin{tabular}{lrrr}
\hline Treatment & Day 8 & Day 15 & Day 29 \\
\hline Occlusive PPT & & & \\
Mapracorat 0.1\% ointment & -43.5 & -70.2 & \multicolumn{1}{c}{-} \\
Prednicarbate ointment 0.25\% & -51.0 & -79.0 & - \\
Vehicle & 4.3 & 0.9 & - \\
Nontreated control (untreated, occluded test field) & 3.0 & 2.4 & - \\
\hline Open PPT & & & \\
Mapracorat 0.1\% ointment & -8.5 & -20.6 & -44.0 \\
Prednicarbate 0.25\% ointment & -20.0 & -32.7 & -50.3 \\
Clobetasol 0.05\% ointment & -43.5 & -64.3 & -88.8 \\
Calcipotriene 0.005\% ointment & -13.5 & -34.9 & -46.3 \\
Calcipotriene 0.005\%/betamethasone $\quad$ & & -82.4 \\
$\quad$ dipropionate 0.05\% ointment & -40.6 & -64.2 & -4 \\
\hline
\end{tabular}

corat $0.1 \%$ ointment (baseline: $369.8 \mu \mathrm{m}$, day 15: 113.3 $\mu \mathrm{m}$ ) and prednicarbate $0.25 \%$ ointment (baseline: 367.9 $\mu \mathrm{m}$, day 15: $89.5 \mu \mathrm{m}$ ) when applied occlusively over a 15day study period (Tables 1, 2; Fig. 1). This corresponded to percent reductions in mean EPB of 70.2 and $79.0 \%$ at the end of the study (day 15), respectively. The comparisons of the mean change from baseline (Table 3) to day 15 showed no meaningful difference between mapracorat $0.1 \%$ ointment and prednicarbate $0.25 \%$ ointment $(-256.6$ vs. $-278.4 \mu \mathrm{m}, p=0.2756)$. In contrast, a clear difference in mean EPB changes from baseline was found between treatment with mapracorat $0.1 \%$ ointment and the corresponding vehicle ointment (-256.6 vs $4.2 \mu \mathrm{m}, p<$ 0.0001). Compared to the nontreated control test field, the vehicle ointment showed only minor changes in the EPB thickness (mean 0.9 vs. $2.4 \%$; Table 1 ).

No meaningful difference in mean EPB changes from baseline was found between the vehicle ointment and the nontreated control $(p=0.8049)$.

\section{Open PPT}

The results of the sonography measurements also showed comparable reductions in mean EPB for mapracorat $0.1 \%$ ointment (baseline: $541.0 \mu \mathrm{m}$, day 15: 427.4 $\mu \mathrm{m}$, day 29: $297.2 \mu \mathrm{m}$ ) and prednicarbate $0.25 \%$ ointment (baseline: $555.7 \mu \mathrm{m}$, day 15: $365.0 \mu \mathrm{m}$, day 29: $281.3 \mu \mathrm{m}$ ) when applied over a 29-day study period (open PPT in Tables 1, 2; Fig. 2). The mean changes from baseline to 
Table 3. Pairwise comparisons of echo-poor band (EPB) thickness (mean change in $\mu \mathrm{m}$ ) measured using 20-MHz B-mode ultrasound in an occlusive $(n=24)$ and open $(n=24)$ psoriasis plaque test $(\mathrm{PPT})$ setting

\begin{tabular}{|c|c|c|c|}
\hline Treatment comparisons & Mean change & $95 \% \mathrm{CI}$ & $p$ value \\
\hline \multicolumn{4}{|l|}{ Occlusive PPT } \\
\hline \multicolumn{4}{|l|}{ Day 15 - baseline } \\
\hline Mapracorat $0.1 \%$ ointment vs. vehicle & -260.8 & $-300.3 /-221.2$ & $<0.0001$ \\
\hline Prednicarbate $0.25 \%$ ointment vs. mapracorat $0.1 \%$ ointment & -21.8 & $-61.3 / 17.8$ & 0.2756 \\
\hline Vehicle vs. nontreated control & -4.9 & $-44.5 / 34.6$ & 0.8049 \\
\hline \multicolumn{4}{|l|}{ Open PPT } \\
\hline \multicolumn{4}{|l|}{ Day 15 - baseline } \\
\hline Mapracorat $0.1 \%$ ointment vs. prednicarbate $0.25 \%$ ointment & 77.1 & $-0.2 / 154.4$ & 0.0507 \\
\hline Mapracorat $0.1 \%$ ointment vs. clobetasol $0.05 \%$ ointment & 255.5 & $178.2 / 332.8$ & $<0.0001$ \\
\hline Mapracorat $0.1 \%$ ointment vs. calcipotriene $0.005 \%$ ointment & 95.1 & $17.8 / 172.4$ & 0.0165 \\
\hline \multicolumn{4}{|l|}{ Mapracorat $0.1 \%$ ointment vs. calcipotriene $0.005 \%$ /betamethasone } \\
\hline dipropionate $0.05 \%$ ointment & 246.1 & $168.8 / 323.4$ & $<0.0001$ \\
\hline \multicolumn{4}{|l|}{ Day 29 - baseline } \\
\hline Mapracorat $0.1 \%$ ointment vs. prednicarbate $0.25 \%$ ointment & 30.6 & $-47.7 / 109.0$ & 0.4396 \\
\hline Mapracorat $0.1 \%$ ointment vs. clobetasol $0.05 \%$ ointment & 247.5 & $169.1 / 325.8$ & $<0.0001$ \\
\hline Mapracorat $0.1 \%$ ointment vs. calcipotriene $0.005 \%$ ointment & 14.8 & $-63.6 / 93.1$ & 0.7086 \\
\hline \multicolumn{4}{|l|}{ Mapracorat $0.1 \%$ ointment vs. calcipotriene $0.005 \%$ /betamethasone } \\
\hline dipropionate $0.05 \%$ ointment & 212.9 & $134.5 / 291.2$ & $<0.0001$ \\
\hline
\end{tabular}

95\% confidence intervals (CI) and 2-sided $p$ values were determined within the framework of the 2-factorial analysis of variance model.

day 29 were -243.8 and $-274.4 \mu \mathrm{m}$, corresponding to reductions of 44.0 and $50.3 \%$, respectively. However, the mean change from baseline to day 15 corresponding to the interval used in the occluded PPT as well as the percent reduction at this time point were lower for mapracorat $0.1 \%$ ointment than prednicarbate $0.25 \%$ ointment ( -113.6 vs. $-190.7 \mu \mathrm{m}, 20.6 \%$ vs. $32.7 \%$ ) in this setting (Tables 1, 2; Fig. 2). The differences in the changes from baseline between these two ointments were $p=0.0507$ and $p=0.4396$ on days 15 and 29 , respectively.

The results following treatment with calcipotriene $0.005 \%$ ointment were similar to those of prednicarbate $0.25 \%$ ointment on days 15 and 29 (baseline: $566.5 \mu \mathrm{m}$, day 15: $357.8 \mu \mathrm{m}$, day 29: $307.9 \mu \mathrm{m}$ ) with changes from baseline of -208.7 on day 15 and $-258.7 \mu \mathrm{m}$ on day 29 , corresponding to percent reductions of 34.9 and $46.3 \%$, respectively. The corresponding $p$ values for the comparisons of calcipotriene $0.005 \%$ ointment with mapracorat $0.1 \%$ ointment on days 15 and 29 were 0.0165 and 0.7086 , respectively.

Greater reductions in EPB were reported for clobetasol $0.05 \%$ ointment (baseline: $557.4 \mu \mathrm{m}$, day 15: $188.3 \mu \mathrm{m}$, day 29: $66.1 \mu \mathrm{m}$ ) and calcipotriene $0.005 \% /$ betamethasone dipropionate $0.05 \%$ ointment (baseline: $552.8 \mu \mathrm{m}$, day 15: $193.0 \mu \mathrm{m}$, day 29: $96.1 \mu \mathrm{m}$ ) on days 15 and 29. The

Comparison of Occlusive and Open

Psoriasis Plaque Tests corresponding mean changes and percent reductions for clobetasol $0.05 \%$ ointment and calcipotriene $0.005 \% / \mathrm{be}$ tamethasone dipropionate $0.05 \%$ ointment compared to baseline were -369.1 and $-359.7 \mu \mathrm{m}$ and 64.3 and $64.2 \%$, respectively, on day 15 , and -491.3 and $-456.7 \mu \mathrm{m}$ and 88.8 and $82.4 \%$, respectively, on day 29 . The $p$ values for the comparisons of the changes from baseline between these two study drugs and mapracorat $0.1 \%$ ointment were $p<0.0001$ each on days 15 and 29, respectively.

\section{Chromametry}

\section{Occluded PPT}

The mean erythema values decreased for mapracorat $0.1 \%$ ointment and prednicarbate $0.25 \%$ ointment during the first 5 days. Thereafter, erythema intensity remained almost constant with no further decrease and no differentiation between mapracorat and prednicarbate. At the end of treatment on day 15 , mean percent reduction in erythema was 34.2 and $34.5 \%$, respectively. No decrease was noted for vehicle and nontreated control (Table 4).

\section{Open PPT}

The mean erythema values decreased following treatment with mapracorat $0.1 \%$ ointment and the other 4 study drugs over the whole study course, yet the highest

Skin Pharmacol Physiol 2017;30:102-114 DOI: $10.1159 / 000458415$ 
Fig. 1. Mean values $( \pm S D)$ of echo-poor band (EPB) thickness measured using 20$\mathrm{MHz} \mathrm{B}$-mode ultrasound (FAS) following occlusive treatment with mapracorat $0.1 \%$, prednicarbate $0.25 \%$, and vehicle ointments, and nontreated control (untreated, occluded test field) in subjects with stable plaque-type psoriasis over a 15-day psoriasis plaque test (PPT). Mapracorat and prednicarbate showed a comparable continuous decrease in mean EPB thickness, whereas only minor changes were observed for the vehicle compared to the negative control.

Fig. 2. Mean values $( \pm S D)$ of echo-poor band (EPB) thickness measured using 20$\mathrm{MHz}$ B-mode ultrasound following open treatment with mapracorat $0.1 \%$, prednicarbate $0.25 \%$, calcipotriene $0.005 \%$, calcipotriene $0.005 \%$ /betamethasone diproprionate $0.05 \%$, and clobetasol $0.05 \%$ ointment in patients with stable plaque-type psoriasis over a 29-day psoriasis plaque test (PPT). Mapracorat showed a similar reduction in mean EPB on day 29 but a lower mean EPB reduction on day 15 compared to prednicarbate. Calcipotriene demonstrated mean EPB decreases similar to those of prednicarbate on days 15 and 29. Highest reductions in mean EPB thickness were observed for clobetasol and calcipotriene/betamethasone dipropionate on days 15 and 29.
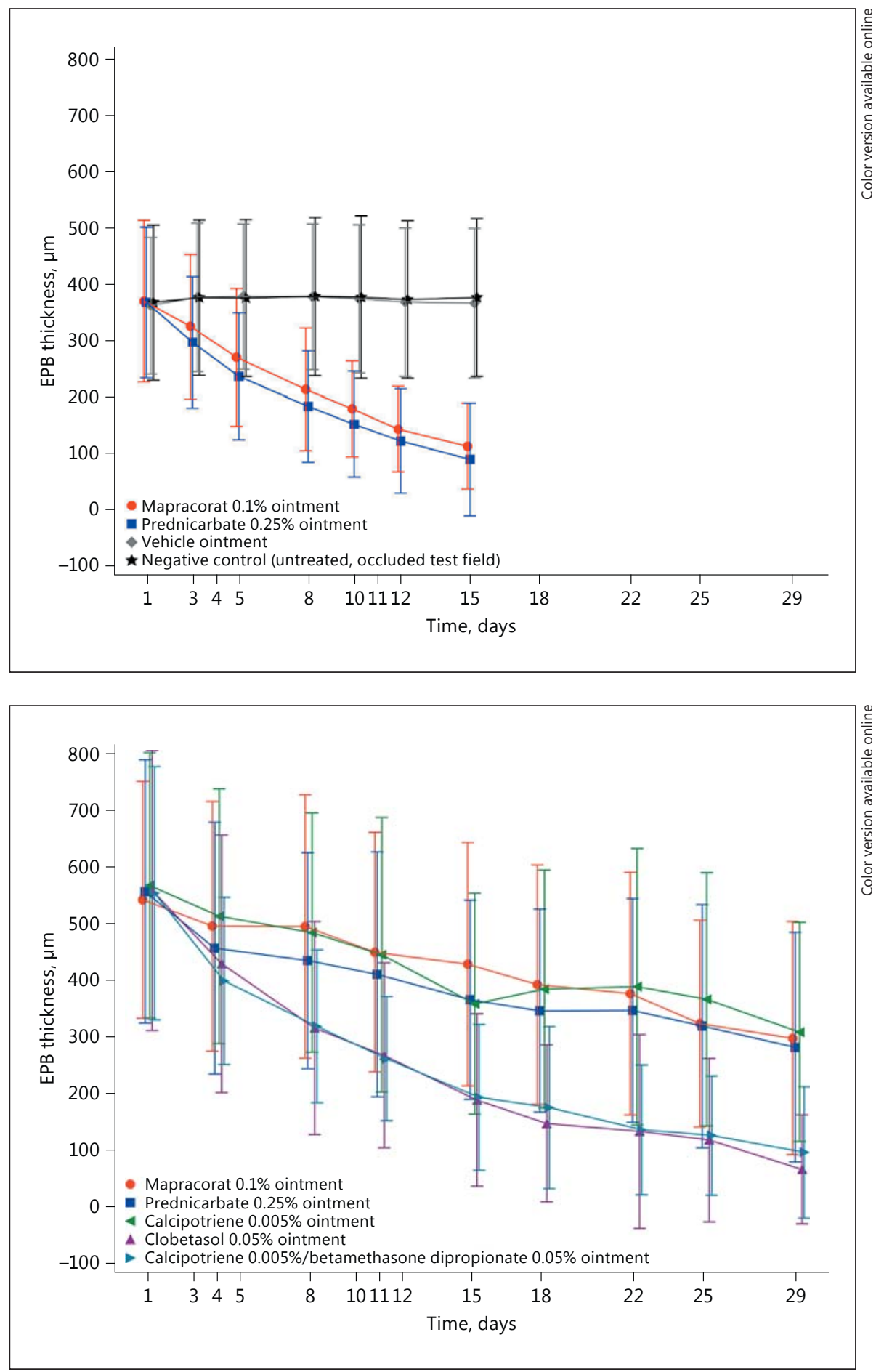

decrease was observed during the first 11 days of treatment. On days 15 and 29, the mean percent reductions in erythema were slightly lower for mapracorat $0.1 \%$ ointment than prednicarbate $0.25 \%$ ointment and calcipotri- ene $0.005 \%$ ointment (day 15: $6.8,13.3$, and $10.4 \%$, respectively; day $29: 8.6,11.6$, and $9.4 \%$, respectively) and considerably lower than for clobetasol $0.05 \%$ ointment and calcipotriene $0.005 \%$ /betamethasone dipropionate 
Table 4. Mean change from baseline in erythema (\%) measured by chromametry in an occlusive $(n=24)$ and open $(n=24)$ psoriasis plaque test (PPT) setting
Table 5. Clinical assessment score (CAS): frequencies (\%) obtained from the occlusive psoriasis plaque test $(\mathrm{PPT})$ $(n=24)$

\begin{tabular}{lrrl}
\hline Treatment & Day 8 & Day 15 & Day 29 \\
\hline Occlusive PPT & & & \\
Mapracorat 0.1\% ointment & -32.8 & -34.2 & - \\
Prednicarbate 0.25\% ointment & -33.2 & -34.5 & - \\
Vehicle & 4.3 & -1.9 & - \\
Nontreated control & 4.2 & -0.5 & - \\
\hline Open PPT & & & \\
Mapracorat 0.1\% ointment & -2.5 & -6.8 & -8.6 \\
Prednicarbate 0.25\% ointment & -4.8 & -13.3 & -11.6 \\
Clobetasol 0.05\% ointment & -16.9 & -24.5 & -29.6 \\
Calcipotriene 0.005\% ointment & -1.6 & -10.4 & -9.4 \\
Calcipotriene 0.005\%/betamethasone & & & \\
$\quad$ dipropionate 0.05\% ointment & -10.8 & -17.0 & -25.1 \\
\hline
\end{tabular}

\begin{tabular}{lcccc}
\hline CAS & $\begin{array}{l}\text { Mapracorat } \\
\text { 0.1\% ointment }\end{array}$ & $\begin{array}{l}\text { Prednicarbate } \\
0.25 \% \text { ointment }\end{array}$ & Vehicle & $\begin{array}{l}\text { Nontreated } \\
\text { control }\end{array}$ \\
\hline Day 15 & & & & \\
-1 & $0(0.0)$ & $0(0.0)$ & $0(0.0)$ & $0(0.0)$ \\
0 & $1(4.2)$ & $0(0.0)$ & $24(100)$ & $23(95.8)$ \\
1 & $8(33.3)$ & $4(16.7)$ & $0(0.0)$ & $1(4.2)$ \\
2 & $14(58.3)$ & $20(83.3)$ & $0(0.0)$ & $0(0.0)$ \\
3 & $1(4.2)$ & $0(0.0)$ & $0(0.0)$ & $0(0.0)$ \\
\hline
\end{tabular}

5-point score: -1 : worsened; 0: unchanged (no effect); 1 : slight improvement; 2 : clear improvement but not completely healed; 3: completely healed.
$0.05 \%$ ointment (day 15: 24.5 and $17.0 \%$, respectively; day 29: 29.6 and $25.1 \%$, respectively) (Table 4).

\section{Clinical Assessment of Efficacy}

In general, the results of the clinical assessments paralleled the findings of the sonographic and chromametric measurements in both PPTs. Worsening (score $=-1$ ) was noted in none of the test fields, neither under occlusive nor under open conditions (for details, the reader is referred to Tables 5, 6).

\section{Safety}

All adverse events were mild or moderate in severity, and had recovered or were recovering at the end of the respective study. None of the adverse events was associated with specific test fields located on psoriasis plaques, and there was no premature study discontinuation based on any adverse event in both studies. All study drugs as well as the procedures performed were well tolerated in the occluded and in the open PPT. There were no serious adverse events in any of the 2 studies.

Comparison of Occlusive and Open

Psoriasis Plaque Tests

\section{Discussion}

The PPT is an experimental clinical model which allows simultaneous topical treatment of several active compounds and their respective vehicles and/or controls intraindividually in the same psoriasis patient. It is a valuable tool to compare and predict treatment efficacy in early clinical development in small cohorts gaining dose and time response curves. In the studies presented, the antipsoriatic effects of mapracorat $0.1 \%$ ointment were investigated compared to established topical products for psoriasis treatment using a classical and a modified PPT which combined the same "objective" measurement techniques. This provided for an ideal setting, enabling us to make data-driven comparisons of the open and the occluded PPT, which, to our knowledge, has not been discussed yet in the extensive literature that exists in this field.

Since its introduction by Dumas and Scholtz [6], the PPT has been published with a variety of test modifications. In many studies in which sonography was per-

Skin Pharmacol Physiol 2017;30:102-114 DOI: $10.1159 / 000458415$ 
Table 6. Clinical assessment score (CAS): frequencies (\%) obtained from the open psoriasis plaque test (PPT) $(n=24)$

\begin{tabular}{cccccc}
\hline & $\begin{array}{l}\text { Mapracorat } \\
\text { 0.1\% ointment }\end{array}$ & $\begin{array}{c}\text { Prednicarbate } \\
0.25 \% \text { ointment }\end{array}$ & $\begin{array}{l}\text { Clobetasol } \\
0.05 \% \text { ointment }\end{array}$ & $\begin{array}{l}\text { Calcipotriene } \\
0.005 \% \text { ointment }\end{array}$ & $\begin{array}{l}\text { Calcipotriene 0.005\%/ } \\
\text { beta-methasone } \\
\text { dipropionate 0.05\% } \\
\text { ointment }\end{array}$ \\
\hline Day 15 & & & & \\
-1 & $0(0.0)$ & $0(0.0)$ & $0(0.0)$ & $0(0.0)$ & $0(0.0)$ \\
0 & $13(54.2)$ & $8(33.3)$ & $2(8.3)$ & $11(45.8)$ & $2(8.3)$ \\
1 & $8(33.3)$ & $11(45.8)$ & $5(20.8)$ & $8(33.3)$ & $6(25.0)$ \\
2 & $2(8.3)$ & $4(16.7)$ & $10(41.7)$ & $5(20.8)$ & $12(50.0)$ \\
3 & $1(4.2)$ & $1(4.2)$ & $7(29.2)$ & $0(0.0)$ & $4(16.7)$ \\
Day 29 & & & & \\
-1 & $0(0.0)$ & $0(0.0)$ & $0(0.0)$ & $0(0.0)$ & $0(0.0)$ \\
0 & $5(20.8)$ & $4(16.7)$ & $0(0.0)$ & $3(12.5)$ & $0(0.0)$ \\
1 & $11(45.8)$ & $13(54.2)$ & $2(8.3)$ & $11(45.8)$ & $2(8.3)$ \\
2 & $5(20.8)$ & $5(20.8)$ & $9(37.5)$ & $10(41.7)$ & $11(45.8)$ \\
3 & $3(12.5)$ & $2(8.3)$ & $13(54.2)$ & $0(0.0)$ & $11(45.8)$ \\
\hline
\end{tabular}

5-point score: -1 : worsened; 0: unchanged (no effect); 1: slight improvement; 2: clear improvement but not completely healed; 3: completely healed.

formed, evaluators measured full skin thickness, also described as distance between the skin surface and the dermis-subcutaneous tissue interface $[14,12,21,26,27]$ and separate measurements of epidermal and dermal thickness [28]. Queille-Roussel et al. [24] measured a value described as "lesion thickness", and in other publications $[23,29,30]$ the parameters "skin thickness" and also "echo-poor band thickness" were evaluated. The observation that the EPB corresponds to the major proportion of the acanthotic epidermis and the superficial dermis is in agreement with findings from el Gammal et al. $[15,16]$. The width of the EPB is not a direct measure of thickness of a psoriasis plaque; however, it reflects the thickness of the papillary dermis, with the degree of vascularity, edema, and inflammation. The thickness of the EPB correlates with the clinical severity of a psoriasis plaque [18].

In general, the three most important disease symptoms of psoriatic skin are thickening (also called induration), erythema, and scaling, and they are also commonly assessed in global disease severity scores. While sonography measurement of the EPB addresses the first aspect and erythema measurement provides an objective parameter for the second, there is no established objective measurement method available to quantify the severity of scaling, so that evaluation commonly relies on clinical grading. In many studies in which sonography measurements were made, scaling was one of several clinical pa- rameters evaluated in addition to sonography [21, 23, 26, 29, 30]. In contrast, Bangha and Elsner [12] described a descaling procedure prior to sonography assessments, and, consequently, scaling was not scored. Dependent on the necessity of carefully removing excessive scales at each measuring time point for optimal ultrasound depth discrimination, this procedure may result in sacrificing scaling as a clinical parameter since an evaluation of this is no longer possible or sensible. The usefulness of the clinical assessment of scales in studies where sonography is the primary assessment tool is debatable. Therefore, to consider all disease-specific symptoms, clinical assessments are still regarded as useful additional design elements in PPTs.

When the PPT was first introduced in the 1970s, occlusion was used to enhance the drug effects that were assessed in this test setting, since it is known that occlusion contributes to the general efficacy of topical drugs, presumably due to promotional effects regarding the skin penetration abilities of pharmacologically active molecules. However, it was only years later that the effects inherent to the occlusion itself, when applied to psoriatic lesions, became widely known. To explain the observed effects of occlusion to psoriatic skin, it was hypothesized that occlusion may lead to a reduction in symptom scores and thickness measurements due to special pharmacodynamic effects of its own [31]. There- 
fore, in the classical occluded test setting, it is generally of interest to test corresponding vehicle and/or nontreated controls, too [7]. To avoid skewing of data, in our experience, an occlusive application test should not exceed 3 weeks.

The focus of the present work was to compare occlusive and open applications in a PPT design, regarding their ability to discriminate between the antipsoriatic activity of different compounds, to assess whether there is consistency between the results obtained with both techniques, and evaluate possible advantages of one design over the other with regard to suitability for use in early drug development. Therefore, we exemplarily used investigations of mapracorat $0.1 \%$ ointment and several approved antipsoriatic topical drugs. Occlusive application is usually chosen to reach a higher level of standardization of the application procedure, to avoid a different grade of environmental (e.g., mechanical) stress to the test sites, and to facilitate the general study conduct, including shorter timelines compared to an open application situation. In contrast, the open PPT is chosen to exclude any occlusion effects that could be regarded as artificial and solely inherent to the test setting, while the goal is to be as consistent as possible with the clinical situation of a later actual psoriasis treatment - despite the early stage of development that usually sets the scene for a PPT.

Overall, in our studies, mapracorat $0.1 \%$ ointment demonstrated a clear antipsoriatic effect that was comparable to the effect seen for prednicarbate $0.25 \%$ ointment both in the occluded and the open PPT, as measured by mean changes in EPB thickness, changes in erythema measurements, as well as clinical assessment scores at the end of treatment compared to baseline. This demonstrates the reproducibility and consistency of results across both test designs. Vehicle and nontreated controls showed no antipsoriatic effect in the occluded PPT. Vice versa, in the open PPT, the substance selected as most potent positive control, clobetasol $0.05 \%$ ointment, showed the expected stronger antipsoriatic effect compared to prednicarbate $0.25 \%$ ointment and mapracorat $0.1 \%$ ointment. The effect of clobetasol $0.05 \%$ ointment was similar to that of calcipotriene $0.005 \% /$ betamethasone dipropionate $0.05 \%$ ointment, another well-established, clinically highly efficacious antipsoriatic product, which was also tested in the open PPT. Thus, overall, analyses of EPB at the end of treatment showed that both the open and the occluded PPT were able to reliably differentiate between the magnitude of antipsoriatic effects of administered treatments. For the occluded PPT, this is in accor-

Comparison of Occlusive and Open

Psoriasis Plaque Tests dance with earlier findings reported by Katz et al. [10] for corticosteroids of different potencies. As expected, the magnitude of the effect of pharmacologically active compounds appeared to be higher in the occluded PPT than the open PPT, despite the shorter treatment duration in the occluded setting.

However, when looking at the dynamics of the EPB changes over time, it appears that there is a somewhat greater difference between prednicarbate $0.25 \%$ ointment and mapracorat $0.1 \%$ ointment in the open PPT than the occluded PPT, especially at earlier time points. In the open PPT, there seems to be a notable difference between these treatments at an early time point that diminishes over the treatment course, with increasing total effect (Table 2). This effect seems not to occur in the occluded PPT setting, where a relatively constant, small delta is observed - with bigger effects being noted already early in the treatment course probably due to the occluded test setting (Table 1). It seems that this phenomenon could represent some kind of ceiling effect, occurring only if the overall effect sizes of the evaluated treatments are large. If so, it would hinder discrimination between treatments and would have to be regarded as a limitation of the occluded PPT that usually results in higher noticeable effect sizes. In effect, such a ceiling effect would reduce the test resolution capacity of EPB changes over time - yet whether this is the case, or whether this observation is just due to statistical scattering of the results, cannot be decided based solely on the present data.

The observed variability in the EPB data seems to be higher in the open PPT than the occluded PPT. This could be easily misinterpreted as supporting the abovediscussed rationale to select an occluded PPT: an assumed higher level of standardization of the application procedure and the avoidance of a different grade of environmental stress to the respective test sites due to the occluded setting might result in a lower variability of the data. However, a closer look at the baseline values reveals that the variability is already higher at baseline in the open $\mathrm{PPT}$, which is most probably rather due to the fact that 2 separately conducted studies are compared than any differences in the procedure. The decreased variability towards the end of treatment observed for the study drugs with the highest respective change in EPB thickness in both studies is most likely due to a floor effect: while some subjects in those groups reach an EPB value of 0 , the downward variability is limited, leading to a lower overall variability in the most effective treatments at the time of their greatest observed effect (day 15 in the occluded PPT and day 29 in the open PPT).

Skin Pharmacol Physiol 2017;30:102-114 DOI: $10.1159 / 000458415$ 
Special caution should be applied in the interpretation of the erythema results. It is likely that the observed effect is caused by a parallel effect of different drug characteristics that may not be specific to antipsoriatic effects, e.g., vasoconstrictive activity. This may be a confounding factor, especially if drug classes are evaluated with this parameter. In contrast, we do see value in adding a clinical assessment in addition to a sonography measurement of the EPB in order to include the important general clinical view into data sets created with this methodology.

In summary, our investigations demonstrated the successful application of both the occluded and the open PPT as efficient, quick, and safe methods to investigate antipsoriatic effects of topical treatments at a very early stage of product development. Both open and occluded application methodologies were able to characterize antipsoriatic effects of topical products inside the frame of the model, with good evidence of consistency of results between the two methods. The occlusive setting had some technical advantages, such as a potentially better standardization and a shorter time of application, resulting in reduced costs. On the other hand, possible advantages of the open setting include closer similarity to later clinical use applications and a potentially higher resolution capacity versus time; however, these come with the downside of a longer treatment period and higher technical challenges regarding the study conduct, requiring sophisticated, established techniques at the study site. The potential benefits of the open design should thus be carefully weighed against the higher efforts.

In summary, the occluded PPT provides an expedient design to evaluate the potential efficacy of candidate drugs but may have limitations in resolution capacity of EPB changes over time, while the open PPT requires a longer application time and is somewhat technically more complex and work intensive, but may be helpful in the evaluation of the antipsoriatic effect of test compounds in early stages of development, mimicking the later clinical use situation with a potentially improved discrimination capacity over the time course of treatment. Therefore, we conclude that in most standard situations, the occluded PPT is a suitable, reliable, and convenient design for screening early development products in psoriasis. While equally valid, the technically more challenging open PPT design should be reserved for development programs in which additional special aspects are focused, such as early investigation of the dynamics of treatment effect over time. The developmental stage of the evaluated products, characteristics of the respective test drugs, as well as any specific objectives of the study need to be considered to come to an informed decision on which design is best suited in a given constellation.

Finally, both PPT models show clear advantages: both can be classified as phase I studies, need only a small number of psoriatic patients due to the intraindividual design in a relatively short study duration, can better discriminate between compounds and concentrations using sonography than clinical assessment alone, enable parallel measurement of treatment response of multiple formulations (dose response and reference products), and allow for the go/no go decision very early in clinical development (often first clinical phase I trial).

\section{Limitations}

Despite the fact that the study setups were similar and both studies were conducted at one investigational site (bioskin ${ }^{\circledR}$, Hamburg, Germany), it should be considered that this was no direct head-to-head comparison. Different subject panels have been tested at different periods, and the drugs chosen for comparison were not standardized between the two studies. While both study populations were comparable to each other due to similar inclusion and exclusion criteria, baseline disease severity regarding mean thickness of EPB differed between the populations, as did baseline variability.

Pretreatment with 5\% salicylic acid was performed similarly in both studies. Its keratolytic effect with increased hydration and softening of the skin potentially might have increased the availability of the subsequently applied topical drugs [32]. Therefore, pretreatment might have influenced the treatment effects or general treatment onset and maximized both test designs as intended for a model.

Finally, in the occluded PPT, only 1 active drug (prednicarbate $0.25 \%$ ointment) was included for comparison, while in the open PPT study, 4 different drugs (prednicarbate $0.25 \%$ ointment, clobetasol $0.05 \%$ ointment, calcipotriene $0.005 \%$ ointment, and calcipotriene $0.005 \%$ / betamethasone dipropionate $0.05 \%$ ointment) with different pharmacological actions and potencies were evaluated for comparison. The comparison of the test designs occluded versus open could, therefore, not be extended to all other antipsoriatic drugs. The test drug was the same in both study settings.
Wigger-Alberti et al. 


\section{Statement of Ethics}

The studies were performed in compliance with local laws and regulations, the Declaration of Helsinki, and the International Conference on Harmonization Good Clinical Practice Guidelines. The study protocols were approved by the relevant independent ethics committee and national health authority. The subjects were investigated after written informed consent was obtained.

\section{Disclosure Statement}

The preparation of this paper was supported by a grant from Bayer AG, Berlin, Germany. W. Wigger-Alberti, R. Williams, Y.L. von Mackensen, and M. Hoffman-Wecker performed and analyzed the trial on a contract research basis. U. Grossmann, G. Staedtler, R. Nkulikiyinka, and K. Shakery are employed by the company that supported this investigation but do not have any personal financial interest in the investigation described in the paper. Data analysis and interpretation were not influenced by the company.

\section{References}

1 Menter A, Korman NJ, Elmets CA, Feldman SR, Gelfand JM, Gordon KB, Gottlieb AB, Koo JY, Lebwohl M, Lim HW, Van Voorhees AS, Beutner KR, Bhushan R: Guidelines of care for the management of psoriasis and psoriatic arthritis: section 4 . Guidelines of care for the management and treatment of psoriasis with traditional systemic agents. J Am Acad Dermatol 2009;61:451-485.

2 Lande R, Botti E, Jandus C, Dojcinovic D, Fanelli G, Conrad C, Chamilos G, Feldmeyer L, Marinari B, Chon S, Vence L, Riccieri V, Guillaume $\mathrm{P}$, Navarini AA, Romero $\mathrm{P}$, Costanzo A, Piccolella E, Gilliet M, Frasca L: The anti-microbial peptide LL37 is a T-cell autoantigen in psoriasis. Nat Commun 2014; 5:5621.

3 Arakawa A, Siewert K, Stöhr J, Besgen P, Kim SM, Rühl G, Nickel J, Vollmer S, Thomas P, Krebs S, Pinkert S, Spannagl M, Held K, Kammerbauer C, Besch R, Dornmair K, Prinz JC: Melanocyte antigen triggers autoimmunity in human psoriasis. J Exp Med 2015;212:22032212.

4 Menter A, Korman NJ, Elmets CA, Feldman SR, Gelfand JM, Gordon KB, Gottlieb A, Koo JY, Lebwohl M, Leonardi CL, Lim HW, Van Voorhees AS, Beutner KR, Ryan C, Bhushan R: Guidelines of care for the management of psoriasis and psoriatic arthritis: section 6 . Guidelines of care for the treatment of psoriasis and psoriatic arthritis: case-based presentations and evidence-based conclusions. J Am Acad Dermatol 2011;65:137-174.

5 Rafael A, Torres T: Topical therapy for psoriasis: a promising future. Focus on JAK and phosphodiesterase-4 inhibitors. Eur J Dermatol 2016;26:3-8.

6 Dumas KJ, Scholtz JR: The psoriasis bio-assay for topical corticosteroid activity. Acta Derm Venereol 1972;52:43-48.

7 Wozel G, Mrowietz U: The psoriasis plaque test on the stage. Acta Derm 1999;99:43-48.

8 Wozel G: Is the psoriasis plaque test still relevant in the age of biologicals? Hautarzt 2006; 57:672-678.
9 Weinstein GD, McCullough JL, Eaglestein WH, Golub A, Cornell RC, Stoughton RB, Clendenning W, Zackheim H, Maibach $\mathrm{H}$, Kulp KR, King L, Baden HP, Tayler JS, Deneau DD: A clinical screening program for topical chemotherapeutic drugs in psoriasis. Arch Dermatol 1981;117:388-393.

10 Katz M, Shaik Z, Maibach HI: Scholtz-Dumas psoriasis small plaque bioassay. J Dermatol Treat 2000;11:15-19.

11 Shargel L, Kanfer I: Generic Drug Product Development: Specialty Dosage Forms. New York, 2010, pp 88-90.

12 Bangha E, Elsner P: Evaluation of topical antipsoriatic treatment by chromametry, visiometry and $20-\mathrm{MHz}$ ultrasound in the psoriasis plaque test. Skin Pharmacol 1996;9:298-306.

13 Savolainen L, Kontinen J, Alatalo E, Röning J, Oikarinen A: Comparison of actual psoriasis surface area and the psoriasis area and severity index by the human eye and machine version methods in following the treatment of psoriasis. Acta Derm Venereol 1998;78:466467.

14 Hoffmann K, el Gammal S, Schwarze H, Dschirka T, Altmeyer P: Examination of psoriasis vulgaris using $20-\mathrm{MHz} \mathrm{B}$-scan ultrasound; in Altmeyer P, el Gammal S, Hoffmann K (eds): Ultrasound in Dermatology. Heidelberg, Springer, 1992, pp 244-249.

15 el Gammal S, Hoffmann K, Auer T, Korten M, Altmeyer P, Höss A, Ermert H: A $50-\mathrm{MHz}$ high resolution ultrasound imaging system for dermatology; in Altmeyer P, el Gammal S, Hoffmann K (eds): Ultrasound in Dermatology. Heidelberg, Springer, 1992, pp 297-322.

16 el Gammal S, Auer T, Popp C, Hoffmann K, Altmeyer P, Passmann C, Ermert H: Psoriasis vulgaris in $50-\mathrm{MHz}$ B-scan ultrasound: characteristic features of stratum corneum, epidermis and dermis. Acta Derm Venereol Suppl (Stockh) 1994;74:173-176.

17 Hoffmann K, Dschirka T, Schwarze H, elGammal S, Matthes U, Hoffmann A, Altmeyer P: $20-\mathrm{MHz}$ sonography, colorimetry and image analysis in the evaluation of psoriasis vulgaris. J Dermatol Sci 1995;9:103-110.
18 Gupta AK, Turnbull DH, Harasiewicz KA, Shum DT, Watteel GN, Foster S, Sauder DN: The use of high-frequency ultrasound as a method of assessing the severity of a plaque of psoriasis. Arch Dermatol 1996;132:658-662.

19 Snape SD, Wigger-Alberti W, Goehring UM: A phase I randomized trial to assess the effect on skin infiltrate thickness and tolerability of topical phosphodiesterase inhibitors in the treatment of psoriasis vulgaris using a psoriasis plaque test. Br J Dermatol 2016;175:479486.

20 Geilen CC, Mrowietz U: Lack of efficacy of topical mycophenolic acid in psoriasis vulgaris. J Am Acad Dermatol 2000;42:837-840.

21 Buder K, Knuschke P, Wozel G: Evaluation of methylprednisolone aceponate, tacrolimus and combination thereof in the psoriasis plaque test using sum score, $20-\mathrm{MHz}$-ultrasonography and optical coherence tomography. Int J Clin Pharmacol Ther 2010;48:814-820.

22 Lee CS, Koo I: The efficacy of three class I topical synthetic corticosteroids, fluocinonide $0.1 \%$ cream, clobetasol $0.05 \%$ cream and halobetasol $0.05 \%$ cream: a Scholtz-Dumas bioassay comparison. J Drugs Dermatol 2009;8: 751-755.

23 Queille-Roussel C, Hoffmann V, Ganslandt C, Hansen KK: Comparison of the anti-psoriatic effect and tolerability of calcipotriol-containing products in the treatment of psoriasis vulgaris using a modified psoriasis plaque test. Clin Drug Investig 2012;32:613-619.

24 Queille-Roussel C, Hoffmann V, Enevold A, Ganslandt C: Use of a psoriasis plaque test in the development of a gel formulation of calcipotriol and betamethasone dipropionate for scalp psoriasis. J Dermatolog Treat 2013;24: 250-254.

25 Barry BW, Woodford R: Comparative bioavailability of proprietary topical corticoid preparations; vasoconstrictor assays on thirty creams and gels. Br J Dermatol 1974;91:323338.
Comparison of Occlusive and Open

Psoriasis Plaque Tests
Skin Pharmacol Physiol 2017;30:102-114 DOI: $10.1159 / 000458415$ 
26 Broby-Johansen U, Karlsmark T, Petersen LJ, Serup J: Ranking of the anti-psoriatic effect of various topical corticosteroids applied under a hydrocolloid dressing - skin-thickness, blood-flow and colour measurements compared to clinical assessments. Clin Exp Dermatol 1990;15:343-348.

27 Lévy J, Gassmueller J, Schroeder G, Audring $\mathrm{H}$, Soennichschen N: Comparison of the effects of calcipotriol, prednicarbate and clobetasol 17-propionate on normal skin assessed by ultrasound measurement of skin thickness. Skin Pharmacol 1994;7:231-236.
28 Cucoş M, Crişan M, Lenghel M, Dudea M, Croitoru R, Dudea SM: Conventional ultrasonography and sonoelastography in the assessment of plaque psoriasis under topical corticosteroid treatment - work in progress. Med Ultrason 2014;16:107-113.

29 Queille-Roussel C, Clonier F, Olesen M: Alternative treatment with calcipotriol plus betamethasone dipropionate gel in psoriasis vulgaris using a short-contact approach. $\mathrm{Br} \mathrm{J}$ Dermatol 2014;171:1596-1598.
30 Queille-Roussel C, Olesen M, Villumsen J, Lacour JP: Efficacy of an innovative aerosol foam formulation of fixed combination calcipotriol plus betamethasone dipropionate in patients with psoriasis vulgaris. Clin Drug Investig 2015;35:239-245.

31 Christophers E, Mrowietz U: The inflammatory infiltrate in psoriasis. Clin Dermatol 1995; 13:131-135.

32 Jacobi A, Mayer A, Augustin M: Keratolytics and emollients and their role in the therapy of psoriasis: a systematic review. Dermatol Ther 2015;5:1-18. 